\title{
Long-term Monitoring with Small and Medium-sized Telescopes on the Ground and in Space
}

\author{
P. A. Charles ${ }^{1,2,3}$, M. M. Kotze $\mathrm{K}^{2,3}$ and A. Rajoelimanana ${ }^{2,3}$ \\ ${ }^{1}$ School of Physics \& Astronomy, University of Southampton, Southampton, UK \\ email: P.A.Charles@soton.ac.uk \\ ${ }^{2}$ South African Astronomical Observatory, Observatory 7935, South Africa \\ ${ }^{3}$ Department of Astronomy, University of Cape Town, Cape Town, South Africa \\ Invited Talk
}

\begin{abstract}
The last 20 years have seen revolutionary developments of large-scale synoptic surveys of the sky, both from the ground (e.g., the MACHO and OGLE projects, which were targetted at micro-lensing studies) and in space (e.g., the X-ray All-Sky Monitor onboard RXTE). These utilised small and medium-sized telescopes to search for transient-like events, but they have now built up a huge database of long-term light-curves, thereby enabling archival research on a wide range of objects that has not been possible hitherto. This is illustrated with examples of long time-scale optical and X-ray variability studies from the field of X-ray binary research: the high-mass BeX binaries in the SMC (using MACHO and OGLE), and the bright galactic-bulge X-ray sources (mostly LMXBs, using RXTE/ASM). As such facilities develop greater capabilities in future and at other wavelengths (developments in South Africa will be described), real-time data processing will allow much more rapid follow-up studies with the new generation of queue-scheduled large telescopes such as SALT.
\end{abstract}

Keywords. telescopes, astronomical data bases: miscellaneous, X-rays: binaries, accretion, accretion disks

\section{Introduction}

The last 20 years have seen remarkable developments in, and expansion of, the number of very large ground-based optical/IR telescopes now available to the global astronomy community (Charles 2011). However, the growth over the last two decades in the number of small $(<2-\mathrm{m})$ telescopes has been on an even larger scale, although such facilities rarely attract high-level media attention. This has occurred even as a number of major observatories have closed or retired many of their smaller telescopes as a result of financial pressures on operating costs. But the latest generation of small telescopes are very different from those older facilities, which had been designed and built as multi-purpose telescopes capable of tackling a wide range of scientific programmes, and whose time was allocated to observers in classical mode (i.e., in blocks of nights or even a week). Instead, the latest small telescopes (and sometimes entire telescope networks) are robotic in operation, usually have a single observing mode, and can be maintained over the Internet. What has made this possible is a consequence of "Moore's Law" in the inexorable rise of computing and electronics performance over the last 30 years, thereby providing automatic telescope control and data processing on scales that would once have been the province of supercomputers but yet are available now for not much more than the cost of a domestic washing machine. Such a setup has removed at a stroke one of the major costs of any telescope operation, namely the provision of local staffing. A parallel factor has 
been the availability of low-cost, panchromatic, large-format CCD detectors, resulting in instruments capable of undertaking programmes that would have been impossible just 20 years ago.

A glimpse of what is possible from dedicated useage of small telescopes (albeit older ones) was provided by the MACHO and OGLE projects beginning in the 1990s (Alcock et al. 1996, Udalski et al. 1997), in their quest for gravitational microlensing events. As a result of the extremely rich star-fields of their main target areas in the Magellanic Clouds and the Galactic Bulge, their archives now provide $>18$-year light-curves of an enormous number of stars.

Among space missions there are analogies of small telescopes that provide generalpurpose survey capabilities, one of the most valuable being the All-Sky Monitor (ASM) on NASA's Rossi X-ray Timing Explorer (RXTE). Since early in 1996, and lasting until the end of 2011, the RXTE/ASM has scanned the entire accessible X-ray sky daily, producing an (immediately available) on-line archive of the light-curves of the bright, mostly Galactic, X-ray sources (Levine et al. 1996).

These ground and space-based facilities have made it possible to study the truly longterm variability of luminous X-ray binaries for the first time. Whilst the spin and orbital periods have been known for many of them ever since their discovery, it is now becoming clear that many, if not all, display so-called "super-orbital" modulations on time-scales of months to years, and that these modulations can occur for different reasons - as summarised by Charles et al. (2010) in their Table 1. These will be used as examples of the kind of science that these monitoring facilities can undertake.

\section{High-Mass X-ray Binaries}

\subsection{BeX systems}

The Be X-ray binaries (BeX systems) are a remarkable population in that they all have neutron-star compact objects (they are pulsars), and approximately half of those known lie in the SMC (Coe 2000). That means that many of them are in the MACHO and OGLE databases, and thus led to the discovery by McGowan \& Charles (2003) of an extraordinary, large-amplitude ( $\sim .5 \mathrm{mag})$ 421-day super-orbital modulation in the optical light-curve of the BeX system A0538-66. Since the 16.6-day orbital period of A0538-66 was well established (on the basis of strictly periodic X-ray and optical flares as the neutron star in its eccentric orbit interacted with the equatorial envelope of the rapidly rotating Be star), McGowan \& Charles (2003) were led to propose that the equatorial envelope (or disk) grew and dissipated on the 421-day timescale.

The MACHO and OGLE databases then provided the opportunity to investigate how widespread such behaviour was amongst the SMC BeX systems, and Fig. 1 (from Rajoelimanana et al. 2011) shows a selection of SMC light-curves from the 50 or so examined. Only a handful display the same behaviour as A0538-66, but that is explained as an inclination effect, with A0538-66 being close to edge-on. Furthermore, many super-orbital (and orbital) modulations were found in this dataset, and Fig. 2 shows how they are correlated. This is to be expected, with the orbit of the compact object restricting the extent to which the equatorial disk of the Be star can expand.

\subsection{Dynamical Power Spectra}

The potential of X-ray monitoring missions to yield remarkable new insights into these long-term physical processes is very well demonstrated in the RXTE/ASM light-curve of SMC X-1 (Fig. 3). This eclipsing HMXB consists of a 0.7-sec X-ray pulsar in a 3.9-day orbit about its B0I companion, which has long demonstrated a $\sim 60$-day super-orbital 

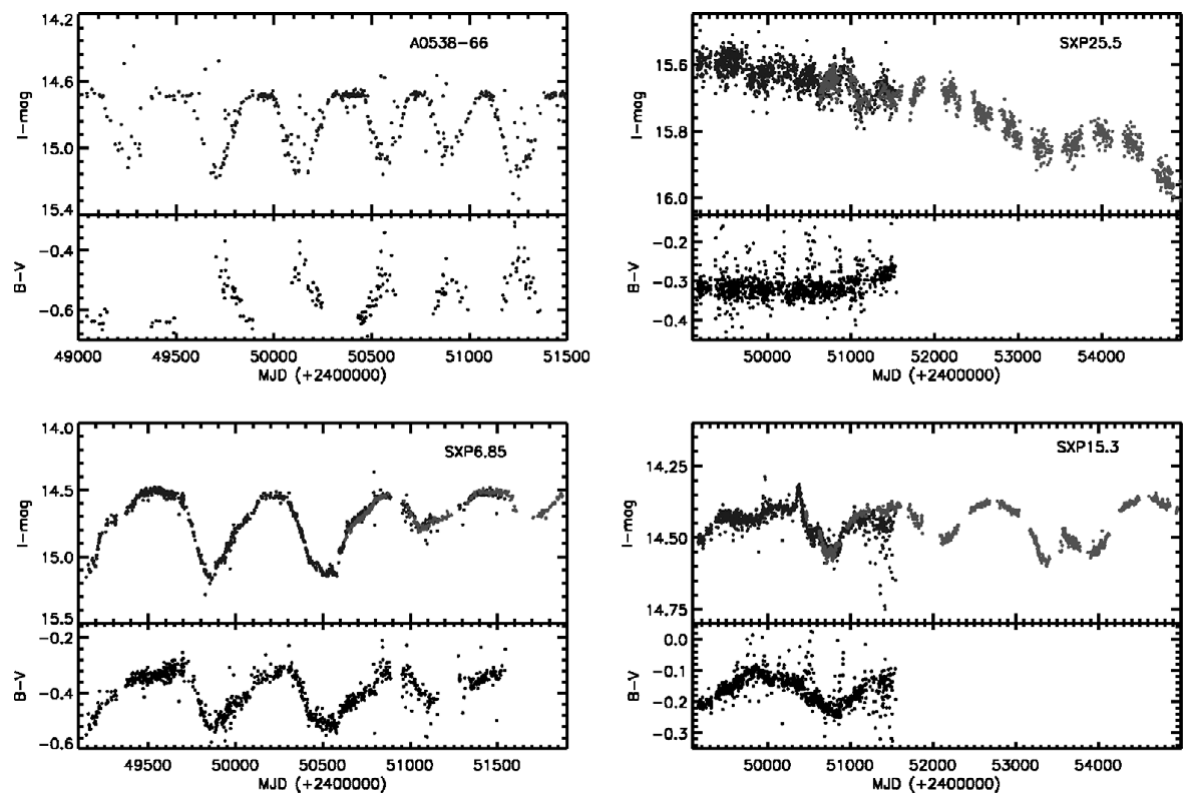

Figure 1. 18-year optical lightcurves of A0538-66 and 3 selected SMC BeX systems that display large-amplitude long-term modulations. (The different colours seen in the on-line version refer to the MACHO (blue), OGLE-I (red) and OGLE-II (green) archives.) From Rajoelimanana et al. (2011).

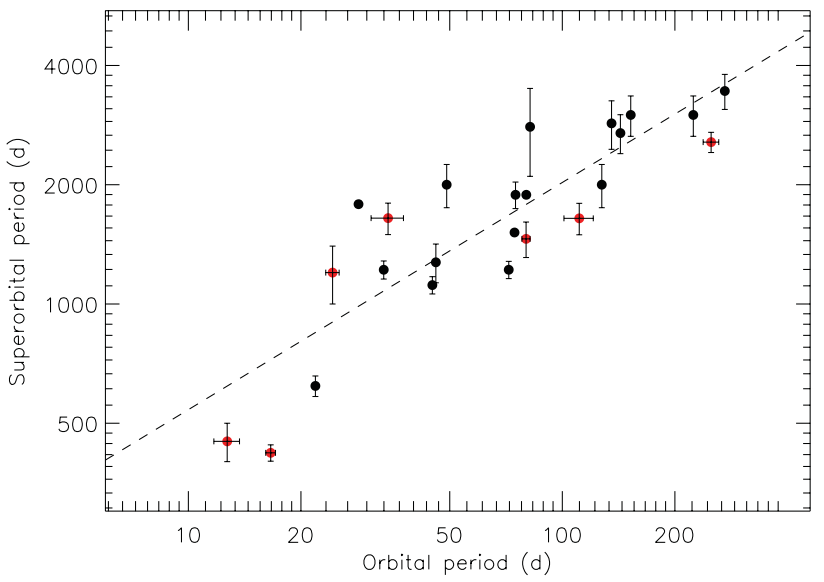

Figure 2. Correlation of super-orbital and orbital periods of SMC BeX systems obtained from the survey of Rajoelimanana et al. (2011), and updated with the results of Reig (2011).

modulation in its X-ray output. However, in the first years of RXTE it became apparent that the period of this modulation was shortening, an observation which led Clarkson et al. (2003) to exploit the long timebase provided by RXTE by applying a "dynamical power spectrum" (DPS) analysis to the SMC X-1 light-curve. The DPS takes a data window of 400 days, derives a periodogram, slides it forward by 50 days, derives a new periodogram, and so on. In this way a time-dependent power spectrum (or DPS) is obtained, which reveals how any super-orbital modulations change on long timescales. That work has been brought up to date by Kotze \& Charles (2011), who utilised the entire RXTE/ASM light-curves of X-ray binaries (for which such modulations have been 


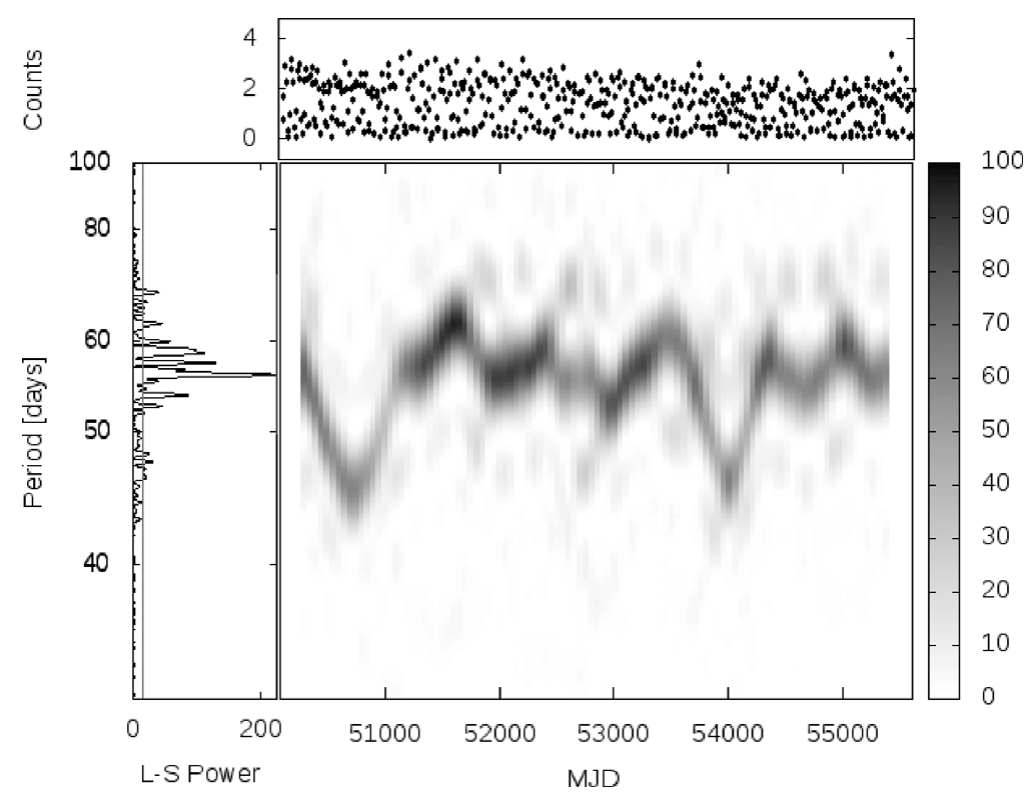

Figure 3. The top box shows the full RXTE/ASM 18-year X-ray light-curve of SMC X-1. The periodogram of the complete light-curve is shown at left in the lower box, and contains multiple significant peaks. The reason for this is demonstrated in the main plot in which the time-dependent periodogram (the Lomb-Scargle power is represented by the grey-scale) reveals how SMC X-1's super-orbital period itself varies with time. From Kotze \& Charles (2011).

reported) to derive their DPS. That of SMC X-1 is shown in Fig. 3, where the time-scale for the drifting of the super-obital modulation is clearly around 5-10 years.

Such super-orbital periods are attributed to a tilted, precessing disk around the compact object, driven by X-ray irradiation. The stability of the disk is a function of the binary mass ratio and separation (Ogilvie \& Dubus 2001). SMC X-1 lies in a region where a warp can be expected to form, leading to the kind of behaviour shown here.

\section{Low-Mass X-ray Binaries}

When Harris et al. (2009) found an almost sinusoidal, 4 year modulation in the RXTE/ASM light-curve of the 4.2-hour LMXB GX9+9, it led Kotze \& Charles (2010) to examine all LMXBs for such very long-term variations. They indeed found similar variations in almost all Atoll and $\mathrm{Z}$ sources, but with the amplitude much greater in the Atoll, compared to the Z, sources. This is demonstrated in Fig. 4 where the correlation with observed average flux levels is very clear. While accurate distances are not known for many of these sources, they are all in the Galactic Bulge (i.e., at distances $\sim 5-$ $12 \mathrm{kpc}$ ), indicating that such behaviour is probably related to the intrinsic luminosity level (since $\mathrm{Z}$ sources are close to $L_{E d d}$, but Atoll sources are $~ 10$ times weaker). Consequently, Kotze \& Charles (2010) have proposed that the modulation is actually due to changes in the mass-transfer rate from the donor, as a result of a solar-cycle-like variation. Similar variations have been seen in CVs, for which this process was first suggested by Applegate \& Patterson (1987). 


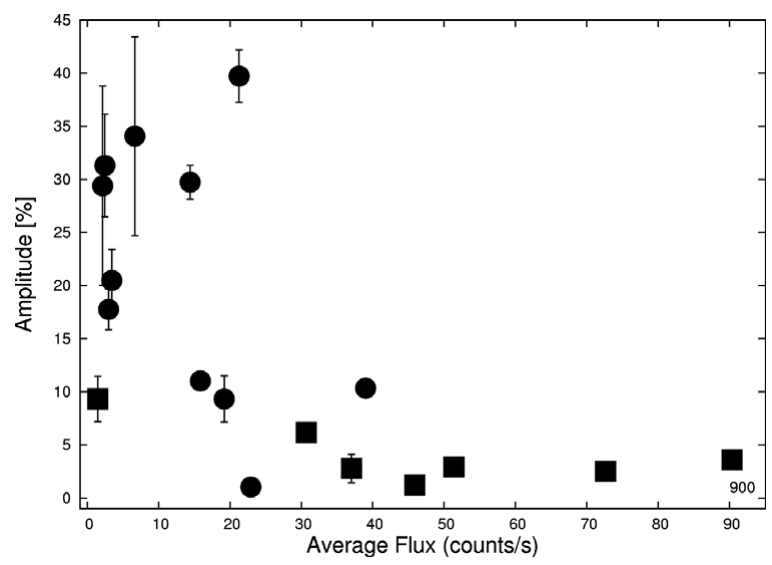

Figure 4. Relationship between the very long-term variability amplitude and average flux levels for Atoll sources (dots) and Z sources (squares), based on the results in Kotze \& Charles (2010).

\section{International Robotic Facilities in South Africa}

The advent of a democratic South Africa has resulted in a dramatic expansion of international telescope projects at the SAAO's observing site (in Sutherland), almost all of them being robotic. They exploit South Africa's "astronomical geographic advantage" (leading to the Sutherland area now being protected by Act of Parliament), but also bring benefits for scientific development within the South African community. The following have all come to pass since 2000:

- IRSF, the IR Survey Facility, a 1.4-m IR telescope (1-2.5 $)$, that began operations in 2000 (Glass \& Nagata 2000). This Japan/South Africa collaborative project conducts IR surveys of the southern skies, concentrating on the Galactic Centre and Bulge regions, as well as the Magellanic Clouds. The results have been the deepest and highest-resolution IR surveys of these regions to date (Kato et al. 2007). IRSF is not robotic, but is operated in classical observing mode, with a mix of Japanese and South African observers.

- SuperWASP, the Wide Angle Search for Planets, is a UK-led project which has been operating robotically at Sutherland since 2005 (there is also a node in La Palma), with the aim of detecting extra-solar planets via observations of transits (Smith et al. 2009). SuperWASP uses large-format CCDs attached to wide-angle lenses to survey the same regions of the sky continuously, reaching $V \sim 14$ out of the Galactic plane, even with $\sim 15$-arcsec pixels. The resulting database of hundreds of billions of observations has revealed more than 30 transiting exoplanets, and is the only such source of bright objects $(V<13)$ in the southern hemisphere; they can then be followed up with highresolution spectroscopy of the parent star. The huge database also provides an archive for independent research programmes, one of which has been determining the detailed properties of contact binaries (Skelton 2010).

- MONET, the MOnitoring NETwork, is a pair of 1.2-m fast-slewing, remotelyoperated telescopes for CCD imaging and fast photometry (Hessman 2001). They are part of the HET/SALT partnership, one being located at the HET site in Texas and the other at Sutherland. They aim to (i) monitor targets to determine their variability state, and hence suitability for related HET/SALT spectroscopy, and (ii) provide a facility for real-time education and outreach work at schools around the world, even involving schools in the real-time operations of the telescope. MONET should begin full operations 
in 2012. Its fast-slewing capability makes it ideal for GRB follow-up, for exoplanet transit timing and for variable star light-curves.

- KELT, the Kilo-degree Extremely Little Telescope, is run by Vanderbilt University and is also a copy of a US-based northern cousin (Kuhn et al. 2010). Similar to SuperWASP in scientific concept, KELT aims to monitor stars brighter than SuperWASP can (in particular $V \sim 8-10 \mathrm{mag}$ ) for transiting exoplanets. Based on a medium-format 35 -mm camera lens attached to a large-format CCD, KELT is low-cost (only $\sim \$ 50 \mathrm{~K}$ ). The commissioning was undertaken collaboratively with UCT.

- KMTNet, the Korean Microlensing Telescope Network, which consists of three identical $\sim 1.8$-m wide-field telescopes to be located in Australia, Chile and South Africa, is to be constructed over the interval 2011-18 and has planet-searching as its prime scientific goal (Kim et al. 2010).

- LCOGT, the Las Cumbres Observatory Global Telescope is a network of (ultimately, at least 6, possibly 8) telescope nodes placed at high-quality sites around the world (Shporer et al. 2011), of which SAAO will be the second main node after Chile (Cerro Tololo). Each node will consist of at least two 1-m telescopes, and at least four 0.4-m telescopes, all of which operate robotically, with the intention of providing continuous all-sky coverage. These telescopes will focus on time-domain astrophysics, having the potential to follow-up GRBs (Gamma-Ray Bursters), supernovæ, microlensing events, planetary transits and a host of other objects rapidly, thereby providing a uniquely powerful facility for research as well as for education.

\section{Acknowledgements}

MK and AR acknowledge bursaries from NASSP and the South African SKA Project.

\section{References}

Alcock, C., et al. 1996, ApJ, 461, 84

Applegate, J. H. \& Patterson, J. 1987, ApJ, 322, L99

Charles, P., Kotze, M., \& Rajoelimanana, A. 2010, AIP Conf. Series, 1314, 303

Charles, P. 2011, in: J.-P. Lasota (ed.), Astronomy at the Frontiers of Science (Dordrecht: Springer), p. 209

Clarkson, W. I., et al. 2003 MNRAS, 339, 447

Coe, M. J. 2000, ASP Conf. Series, 214, 656

Glass, I. S. \& Nagata, T. 2000, MNASSA, 59, 110

Harris, R. J., et al. 2009 ApJ, 696, 1987

Hessman, F. V. 2001, ASP Conf.Series, 246, 13

Kato, D., et al. 2007, PASJ, 59, 615

Kim, S.-L., et al. 2010, SPIE, 7733, 107

Kotze, M. M. \& Charles, P. A. 2010, MNRAS, 402, L16

Kotze, M. M. \& Charles, P. A. 2011, MNRAS, online

Kuhn, R., et al. 2010, BAAS, 42, 288

Levine, A.M., et al. 1996, ApJ, 469, L33

McGowan, K. E. \& Charles, P. A. 2003, MNRAS, 339, 748

Ogilvie, G. I. \& Dubus, G. 2001, MNRAS, 320, 485

Rajoelimanana, A. F., Charles, P. A., \& Udalski, A. 2011, MNRAS, 413, 1600

Reig, P. 2011, Ap.Sp.Sci., 332, 1

Shporer, A., et al. 2011, in: A. Sozzetti, M. G. Lattanzi \& A. P. Boss (eds.), The Astrophysics of Planetary Systems, IAU Symp. 276, Cambridge, UK: Cambridge University Press, p. 553

Skelton, P. 2010, in: I. Basson \& A. E. Botha (eds.), Modelling of Eclipsing Binaries, $55^{\text {th }}$ SAIP Ann. Conf., (Pretoria: CSIR)

Smith, A. M. S., et al. 2009, MNRAS, 398, 1827

Udalski, A., Kubiak, M., \& Szymanski, M. 1997, Acta Ast., 47, 319 